\title{
Deceleration Parameter $Q(Z)$ and Examining If a Joint DM-DE Model Is Feasible, with a Revisit to the Question of Cosmic Singularities
}

\author{
Andrew Beckwith \\ Department of Physics, Chongqing University, Chongqing, China \\ Email: abeckwith@uh.edu,rwill9955b@gmail.com
}

Received 29 April 2016; accepted 4 July 2016; published 8 July 2016

Copyright (C) 2016 by author and Scientific Research Publishing Inc.

This work is licensed under the Creative Commons Attribution International License (CC BY). http://creativecommons.org/licenses/by/4.0/

c) (†) Open Access

\begin{abstract}
This paper is a revisit to a 2011 document, with the addition of results pertinent to singularities in the case of a single repeating universe, as well as when the multiverse voids the necessity of a classical GR singularity. When a classical singularity does not exist, it impacts the formation of a massive graviton for reasons brought up, and allows for reacceleration of the universe due to massive gravitons. The existence of massive gravitons would also affect initial entropy, and also lead to the datum, that a calculated inflaton $\phi(t)$ may re-emerge after fading out in the aftermath of inflation. The inflaton may be a contributing factor, with non-zero graviton mass, in reacceleration of the universe a billion years ago. The inflaton is a source of reacceleration of the universe, especially if the effects of a re-emergent inflaton are in tandem with the appearance of macro effects of a small graviton mass, leading to a speed up of the rate of expansion of the universe one billion years ago, at red shift value of $Z \sim 0.423$. We find that the graviton being massless or massive directly affects graviton contributions to reacceleration of the universe, with other phenomenological consequences. Finally we give our own counterpart as to how much space-time should be transferred to the present cosmological inflationary cycle which may permit preservation of Planks constant value and support Corda's brilliant "gravity's breath" document.
\end{abstract}

\section{Keywords}

Inflaton, Non Zero Graviton Mass, Emergent Structure, BBN, Singularities

\section{Introduction}

We begin with a brief model as to singular universe, versus a multiverse in terms of input into singularity con-

How to cite this paper: Beckwith, A. (2016) Deceleration Parameter $Q(Z)$ and Examining If a Joint DM-DE Model Is Feasible, with a Revisit to the Question of Cosmic Singularities. Journal of High Energy Physics, Gravitation and Cosmology, 2, 362-382. http://dx.doi.org/10.4236/jhepgc.2016.23033 
struction. The singularity behavior envisioned in this document is given by the following argument, as given by Kauffman [1] and the author in [2], with the case of when one has been reevaluating the question of a "near singularity" in a multiverse. The multiverse will assume Ergotic mixing of space-time as given by [3]. Massless gravitons corresponds to the physics described in the beginMassless gravitons corresponds to the physics described in the beginning of the 2nd part of this document, whereas if massive gravitons exist, the resulting alterations of the general relativistic equations will be linking us to review questions as to if singularities exist at the start of cosmological expansion. Or, the start of cosmological expansions, if massive gravitons exist, assumes the existence of a small non singular regime of space-time. This sharply differentiates us from the physics given in reference [4], as we explain in our document.

\section{Review of the Formalism of Congruence or Lack of with Singularities If a Massive Graviton Exists, in Early Universe Geometry}

We follow the recent work of Kauffmann [1], which sets an upper bound to concentrations of energy, in terms of how he formulated the following equation put in below as Equation (1). Equation (1) specifies an inter-relationship between an initial radius $R$ for an expanding universe, and a "gravitationally based energy" expression we will call $T_{G}(r)$ which lead to a lower bound to the radius of the universe at the start of the Universe's initial expansion, with manipulations. The term $T_{G}(r)$ is defined via (2) afterwards. We start off with Kauffmann's expression [1]

$$
R \cdot\left(\frac{c^{4}}{G}\right) \geq \int_{\left|r^{\prime \prime}\right| \leq R} T_{G}\left(r+r^{\prime \prime}\right) \mathrm{d}^{3} r^{\prime \prime}
$$

Kauffmann [1] calls $\left(\frac{c^{4}}{G}\right)$ a "Planck force” which is relevant due to the fact we will employ (1) at the initial instant of the universe, in the Planckian regime of space-time. Also, we make full use of setting for small $r$, the following:

$$
T_{G}\left(r+r^{\prime \prime}\right) \approx T_{G=0}(r) \cdot \text { const } \sim V(r) \sim m_{\text {Gravition }} \cdot n_{\text {Initial-entropy }} \cdot c^{2}
$$

i.e. what we are doing is to make the expression in the integrand proportional to information leaked by a past universe into our present universe, with $\mathrm{Ng}$ [5]-[18] style quantum infinite statistics use of

$$
n_{\text {Initial-entropy }} \sim S_{\text {Gravition-count-entropy }}
$$

Then Equation (3) will lead to

$$
\begin{aligned}
R \cdot\left(\frac{c^{4}}{G}\right) & \geq \int_{\left|r^{\prime \prime}\right| \leq R} T_{G}\left(r+r^{\prime \prime}\right) \mathrm{d}^{3} r^{\prime \prime} \approx \text { const } \cdot m_{\text {Gravition }} \cdot\left[n_{\text {Initial-entropy }} \sim S_{\text {Gravition-count-entropy }}\right] \\
& \Rightarrow R \cdot\left(\frac{c^{4}}{G}\right) \geq \text { const } \cdot m_{\text {Gravition }} \cdot\left[n_{\text {Initial-entropy }} \sim S_{\text {Gravition-count-entropy }}\right] \\
& \Rightarrow R \geq\left(\frac{c^{4}}{G}\right)^{-1} \cdot\left[\text { const } \cdot m_{\text {Gravition }} \cdot\left[n_{\text {Initial-entropy }} \sim S_{\text {Gravition-count-entropy }}\right]\right]
\end{aligned}
$$

Here, $\left[n_{\text {Initial-entropy }} \sim S_{\text {Gravition-count-entropy }}\right] \sim 10^{5}, m_{\text {Gravition }} \sim 10^{-62}$ grams, and we set Planck length as:

$$
\text { Planck length }=l_{\text {Planck }}=1.616199 \times 10^{-35} \text { meters }
$$

where we set $l_{\text {Planck }}=\sqrt{\frac{\hbar G}{c^{3}}}$ with $R \sim l_{\text {Planck }} \cdot 10^{\alpha}$, and $\alpha>0$. Typically $R \sim l_{\text {Planck }} \cdot 10^{\alpha}$ is about $10^{3} \cdot l_{\text {Planck }}$ at the outset, when the universe is the most compact. The value of const is chosen based on common assumptions about contributions from all sources of early universe entropy, and will be more rigorously defined in a later paper. We argue that the above methodology, giving a non zero initial starting point is made especially tend ible if one is using a low temperature start, allowing for the existence of prior recycling universes gravitons to play a role, i.e. 
that in the single universe repeated again and again, there would be real issues as to the survival of the graviton allowing for the conclusion as to Equation (4). What Equation (4) is doing is to help us determine if conditions exist for a massive graviton versus a massless graviton. If Equatuion (4) is consistent with the existence of massive gravitons, then our inflaton model contributes to models which have Dark Energy as due directly to the existence of massive gravitons in space-time.

\section{Looking at Measuring Gravity Waves, and Gravitons, with Mass}

We will start with a first-principle introduction to detection of gravitational wave density using the definition given by Maggiore [6]

$$
\Omega_{g w} \equiv \frac{\rho_{g w}}{\rho_{c}} \equiv \int_{f=0}^{f=\infty} \mathrm{d}(\log f) \cdot \Omega_{g w}(f) \Rightarrow h_{0}^{2} \Omega_{g w}(f) \cong 3.6 \cdot\left[\frac{n_{f}}{10^{37}}\right] \cdot\left(\frac{f}{1 \mathrm{kHz}}\right)^{4}
$$

where $n_{f}$ is the frequency-based numerical count of gravitons per unit phase space. The author suggests that $n_{f}$ may also depend upon the interaction of gravitons with neutrinos in plasma during early-universe nucleation, as modeled by M. Marklund et al. [7]. Having said that, the question is, what sort of mechanism is appropriate for considering macro effects of gravitons, and the author thinks that he has one, i.e. reacceleration of the universe, as far as a function of graviton mass, i.e. what Beck with is to modify is what was in reference [8] Assume Snyder geometry and look at use of the following inequality for a change in the HUP [8],

$$
\Delta x \geq\left[(1 / \Delta p)+l_{s}^{2} \cdot \Delta p\right] \equiv(1 / \Delta p)-\alpha \cdot \Delta p
$$

and that the mass of the graviton is partly due to the stretching alluded to by Fuller and Kishimoto [9] a supposition the author is investigating for a modification of a joint KK tower of gravitons, as given by Maartens [10] [11] for DM. Assume the stretching of early relic neutrinos that would lead to the KK tower of gravitons-for when $\alpha<0$, is,

$$
m_{n}(\text { Graviton })=\frac{n}{L}+10^{-65} \text { grams }
$$

Note that Rubakov [12]-[14] writes KK graviton representation as, after using the following normalization $\int \frac{\mathrm{d} z}{a(z)} \cdot\left[h_{m}(z) \cdot h_{\tilde{m}}(z)\right] \equiv \delta(m-\tilde{m})$ where $J_{1}, J_{2}, N_{1}, N_{2}$ are different forms of Bessel functions, to obtain the KK graviton/DM candidate representation along RS dS brane world [12]

$$
h_{m}(z)=\sqrt{m / k} \cdot \frac{J_{1}(m / k) \cdot N_{2}([m / k] \cdot \exp (k \cdot z))-N_{1}(m / k) \cdot J_{2}([m / k] \cdot \exp (k \cdot z))}{\sqrt{\left[J_{1}(m / k)\right]^{2}+\left[N_{1}(m / k)\right]^{2}}}
$$

This Equation (8) and Equation (9) is for $\mathrm{KK}$ gravitons having a $\mathrm{TeV}$ magnitude mass $M_{Z} \sim k$ (i.e. for mass values at. $5 \mathrm{TeV}$ to above a $\mathrm{TeV}$ in value) on a negative tension RS brane. What would be useful would be managing to relate this KK graviton, which is moving with a speed proportional to $H^{-1}$ with regards to the negative tension brane with $h \equiv h_{m}(z \rightarrow 0)=$ const $\cdot \sqrt{\frac{m}{k}}$ as an initial starting value for the KK graviton mass, before the KK graviton, as a "massive" graviton moves with velocity $H^{-1}$ along the RS dS brane. If so, and if $h \equiv h_{m}(z \rightarrow 0)=$ const $\cdot \sqrt{\frac{m}{k}}$ represents an initial state, then one may relate the mass of the KK graviton, moving at high speed, with the initial rest mass of the graviton, which in four space in a rest mass configuration would have a mass lower in value, i.e. of $m_{\text {graviton }}(4$-Dim GR $) \sim 10^{-48} \mathrm{eV}$, as opposed to $M_{X} \sim M_{\text {KK-Graviton }} \sim 0.5 \times 10^{9} \mathrm{eV}$. Whatever the range of the graviton mass, it may be a way to make sense of what was presented by Dubovsky et al. [15] who argue for graviton mass using CMBR measurements, of $M_{\text {KK-Graviton }} \sim 10^{-20} \mathrm{eV}$ Dubosky et al. [15] results can be conflated with Alves et al. [16] arguing that non zero graviton mass may lead to an acceleration of our present universe, in a manner usually conflated with $\mathrm{DE}$, i.e. their graviton mass would be about $m_{\text {graviton }}(4$-Dim GR $) \sim 10^{-48} \times 10^{-5} \mathrm{eV} \sim 10^{65}$ grams. Also assume that to calculate the deceleration, the following 
modification of the HUP is used: [2] $\Delta x \geq\left[(1 / \Delta p)+l_{s}^{2} \cdot \Delta p\right] \equiv(1 / \Delta p)-\alpha \cdot \Delta p$, where the LQG condition is $\alpha>0$, and brane worlds have, instead, $\alpha<0$ Also (10) will be the starting point used for a KK tower version of (10) below. So from Maarten's [10] [11] paper,

$$
\dot{a}^{2}=\left[\left(\frac{\tilde{\kappa}^{2}}{3}\left[\rho+\frac{\rho^{2}}{2 \lambda}\right]\right) a^{2}+\frac{\Lambda \cdot a^{2}}{3}+\frac{m}{a^{2}}-K\right]
$$

Maartens [10] [11] also gives a $2^{\text {nd }}$ Friedman equation, as

$$
\dot{H}^{2}=\left[-\left(\frac{\tilde{\kappa}^{2}}{2} \cdot[p+\rho] \cdot\left[1+\frac{\rho^{2}}{\lambda}\right]\right)+\frac{\Lambda \cdot a^{2}}{3}-2 \frac{m}{a^{4}}+\frac{K}{a^{2}}\right]
$$

Also, we are in the regime for which $\rho \cong-P$, for redshift values $z$ between zero and $1.0-1.5$ with exact equality of pressure being equal to the negative value of density, $\rho=-P$, for redshift $z$ between zero to 0.5 . The net effect will be to obtain, due to (6), and use $a \equiv\left[a_{0}=1\right] /(1+z)$. As given by Beckwith [17] [18]

$$
q=-\frac{\ddot{a} a}{\dot{a}^{2}} \equiv-1-\frac{\dot{H}}{H^{2}}=-1+\frac{2}{1+\tilde{\kappa}^{2}[\rho / m] \cdot(1+z)^{4} \cdot(1+\rho / 2 \lambda)} \approx-1+\frac{2}{2+\delta(z)}
$$

Equation (12) assumes $\Lambda=0=K$, and the net effect is to obtain, a substitute for DE, by presenting how gravitons with a small mass done with $\Lambda \neq 0$, even if curvature $K=0$.

\section{Consequences of Small Graviton Mass for Reacceleration of the Universe}

In a revision of Alves et al. [16], Beckwith [17] [18] used a higher-dimensional model of the brane world and Marsden [10] [11] KK graviton towers. The density $\rho$ of the brane world in the Friedman equation as used by Alves et al. [16] is use by Beckwith for a non-zero graviton [17] [18]

$$
\rho \equiv \rho_{0} \cdot(1+z)^{3}-\left[\frac{m_{g} \cdot(c=1)^{6}}{8 \pi G(\hbar=1)^{2}}\right] \cdot\left(\frac{1}{14 \cdot(1+z)^{3}}+\frac{2}{5 \cdot(1+z)^{2}}-\frac{1}{2}\right)
$$

i.e. Equation (12), and Equation (13) above is making a joint DM and DE model, with all of. (13) being for KK gravitons and DM, and $10^{-65}$ grams being a 4 dimensional DE. (11) is part of a KK graviton presentation of DM/DE dynamics. Beckwith [17] [18] found at $z \sim 0.4$, a billion years ago, that acceleration of the universe increased, as shown in Figure 1 [17] [18].

\section{What If an Inflaton Partly Re-Emerges in Space-Time Dynamics? At z 0.423?}

Padmanabhan [19] [20] has written up how the $2^{\text {nd }}$ Friedman equation as of (11), which for $z \sim 0.423$ may be simplified to read as [10] [11]

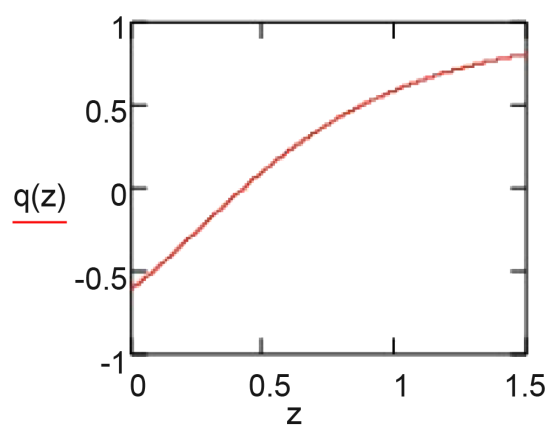

Figure 1. Reacceleration of the universe based on Beckwith [17] [18] (note that $q<0$ if $z<0.423$ ). 


$$
\dot{H}^{2} \cong\left[-2 \frac{m}{a^{4}}\right]
$$

Equation (14) would lead to an inflaton value of, when put in, for scale factor behavior as given by $a(t) \propto t^{\lambda}, \lambda=(1 / 2)-\varepsilon^{+}, 0 \leq \varepsilon^{+} \ll 1$, of, for the inflatonand inflation of [19] [20]

$$
\phi(t)=\int \mathrm{d} t \cdot \sqrt{-\frac{\dot{H}}{4 \pi G}}
$$

Assuming a decline of $a(t) \propto t^{\lambda}, \lambda=(1 / 2)-\varepsilon^{+}, 0 \leq \varepsilon^{+} \ll 1$, Equation (15) yields [19]

$$
\phi(t) \sim \sqrt{\frac{2 m}{4 \pi G}} \cdot\left[2 \varepsilon^{+}\right] \cdot t^{2 \cdot \varepsilon^{+}}
$$

As the scale factor of $a(t) \propto t^{\lambda}, \lambda=(1 / 2)-\varepsilon^{+}, 0 \leq \varepsilon^{+} \ll 1$ had time of the value of roughly $a(t) \propto t^{\lambda}, \lambda=(1 / 2)-\varepsilon^{+}, 0 \leq \varepsilon^{+} \ll 1$ have a power law relationship drop below $a(t) \propto t^{1 / 2}$, the inflaton took Equation (16)'s value which may have been a factor as to the increase in the rate of acceleration, as noted by the q factor, given in Figure 1. Note that there have been analytical work projects relating the inflaton, and its behavior to entropy via noting that inflation stopped when the inflaton field settled down into a lower lower energy state. The way to relate an energy state to the inflaton is, if $a(t)=a_{0} t^{\lambda}$, then in the early universe, one has a potential energy term of [19] [20]

$$
V(\phi)=V_{0} \cdot \exp \left[-\sqrt{\frac{16 \pi G}{\lambda}} \cdot \phi(t)\right]
$$

A situation where both $\lambda=(1 / 2)-\varepsilon^{+}$grows smaller, and, temporarily, $\phi(t)$ takes on Equation (16)'s value, even if the time value gets large, and also, if acceleration of the cosmic expansion is taken into account, then there is infusion of energy by an amount $\mathrm{d} V$. The entropy $\mathrm{d} S \simeq \mathrm{d} V / T$, will lead, if there is an increase in $V$, as given by Equation (17) a situation where there is an effective increase in entropy. If there is, as will be related to later, circumstances, where [5] $S \approx N=$ number of graviton states [17] [18] as will be derived in Equation (17), then at least in higher dimensions, we have an argument that the re emergence of an inflaton, with a corresponding reduction of Equation (17) in magnitude may be part of gravitons playing a role in the re acceleration of the universe.

\section{Other than Five Dimensions for Cosmology? Problems Which Need Resolutions}

If a way to obtain a graviton mass in four dimensions is done which fits in with the as given higher 5 dimensions specified by a slight modification of brane theory, or Maarten's cosmological evolution [10] [11] equations, what benefits could this approach accrue for other outstanding problems in cosmology? The author, Beckwith, claims that due to the Friedmann equations, it would result in deceleration parameter $q(\mathrm{z})$ similar to Figure 1 above. Snyder geometry for the four dimensional case with would specify Friedmann equations along the lines of $\alpha>0$ in Equation (2) above. If one follows $\alpha<0$, then the Friedmann equations appear as giving details to the following equation [21]

$$
\mathfrak{I}=-\frac{1}{2} \int \mathrm{d}^{4} x h_{u v}^{0} \cdot T^{u v} \sim L^{2} \approx \delta^{+} \geq 0
$$

The construction done from sections 1 to 3 are for $\alpha<0$. When $\alpha>0$, the claim is that almost all the complexity is removed $\alpha>0$, and what is left is a [21] treatment of the Friedmann equations, where he obtains, to first order, if $\rho$ is a scalar field density,

$$
\left(\frac{\dot{a}}{a}\right)^{2}=[\kappa / 3] \cdot \rho
$$

and

$$
\left(\frac{\ddot{a}}{a}\right)=-[2 \cdot k / 3] \cdot \rho
$$


The interpretation of $\rho$ as a scalar field density [21], and if one does as Alves et al. [16] uses Equation (7) above. We need to interpret the role of $\rho$. In the LQG version, Equation (20) may be rewritten as follows: If conjugate momentum is in many cases, "almost" or actually a constant, using $\phi=-[\hbar / i] \cdot\left[\partial / \partial p_{\phi}\right]$

$$
\left(\frac{\dot{a}}{a}\right)^{2} \equiv[\kappa / 6] \cdot\left[p_{\phi}^{2} / a^{6}\right]
$$

Beckwith [17] [18] claims that the deceleration parameter q(z) incorporating Equation (19), Equation (20) and. Equation (21) should give much the same behavior as Figure 1 above. If so, then if one is differentiating between four and five dimensions by what is gained, in cosmology, one needs having it done via other criteria. The following is a real problem. As given by Maggiore [6], the massless equation of the graviton evolution equation takes the form

$$
\partial_{\mu} \partial^{\omega} h_{\mu \nu}=\sqrt{32 \pi G} \cdot\left(T_{\mu \nu}-\frac{1}{2} \eta_{\mu v} T_{\mu}^{\mu}\right)
$$

When $m_{\text {graviton }} \neq 0$, the above becomes [6]

$$
\left(\partial_{\mu} \partial^{\sigma}-m_{\text {graviton }}\right) \cdot h_{\mu v}=\left[\sqrt{32 \pi G}+\delta^{+}\right] \cdot\left(T_{\mu v}-\frac{1}{3} \eta_{\mu v} T_{\mu}^{\mu}+\frac{\partial_{\mu} \partial_{v} T_{\mu}^{\mu}}{3 m_{\text {graviton }}}\right)
$$

The mismatch between these two equations, when $m_{\text {graviton }} \rightarrow 0$, is due to $m_{\text {graviton }} h_{\mu}^{\mu} \neq 0$ as $m_{\text {graviton }} \rightarrow 0$, which is due to setting a value of $m_{\text {graviton }} \cdot h_{\mu}^{\mu}=-\left[\sqrt{32 \pi G}+\delta^{+}\right] \cdot T_{\mu}^{\mu}$ The semi classical method by t'Hooft [22] [23], using Equation (22) and Equation (23) is the solution. We generalize to higher dimensions the following diagram as given by Beckwith [24] [25]. Use an instanton- anti instanton structure, and t'Hooft [22] [23] equivalence classes along the lines of (24) below with equivalence class structure in the below wave functional to be set by a family of admissible values [24] [25] $\phi_{0}(x)$

$$
\left.\Psi_{i, f}[\phi(x)]\right|_{\phi \equiv \phi_{c, i, f}}=c_{i, f} \cdot \exp \left\{-\int \mathrm{d} \boldsymbol{x} \alpha\left[\phi_{C i, f}(\boldsymbol{x})-\phi_{0}(\boldsymbol{x})\right]^{2}\right\}
$$

We state that the process of nucleation of a graviton at the initiation of space-time creation. is similar in part to what occurs in the instanton-anti instanton formulation of Figure 2, above. At the end of the document will be a supposition as to taking this analogy far more directly as to the nature of gravitons, as a future works project.

This discussion above, would be consistent upon having a graviton represented by not only Equation (24). If one is adding the small mass of $m_{n}($ Graviton $)=\frac{n}{L}+10^{-65}$ grams, with $m_{0}($ Graviton $) \approx 10^{-65}$ grams, then the problem being worked with is a source term problem of the form given by Peskins [26] as of the type

$$
\psi_{n}(x) \equiv \int \mathrm{d}^{3} p \cdot \frac{1}{(2 \pi)^{3}} \cdot \frac{1}{\sqrt{2 E_{P}}} \cdot\left\{\left(a_{P}+\frac{i}{\sqrt{2 E_{P}}} \cdot F T\left(m_{0}(\text { graviton })\right)\right) \exp (-i p x)+\text { H.C. }\right\}
$$

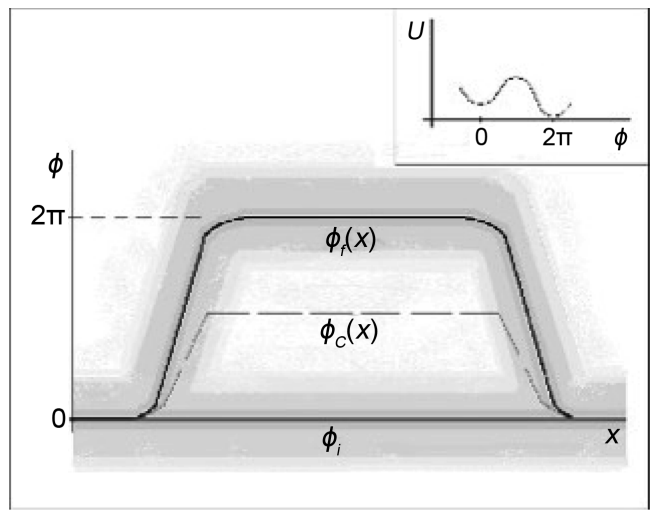

Figure 2. The pop up effects of an intanton-anti-instanton in euclidian space [24] [25]. 
This is, using the language Rubakov [12] put up equivalent to obtain,

$$
\psi_{m}(x) \approx h_{m}(x)+\int \mathrm{d}^{3} p \cdot \frac{1}{(2 \pi)^{3}} \cdot\left(\frac{1}{\sqrt{2 E_{P}}}\right)^{2} \cdot\left\{\left(i \cdot F T\left(m_{0}(\text { graviton })\right)\right) \exp (-i p x)+H . C .\right\}
$$

If $m_{0}$ (graviton) is a constant, then the expression (24b) has delta functions. This is the field theoretic identification. Another way is to consider an instanton-anti instanton treatment of individual gravitons, and to first start with the supposed stretch out of gravitons to enormous lengths. Assuming $m_{0}$ (Graviton) $\approx 10^{-65}$ grams for gravitons in 4 dimensions, the supposition by Bashinsky [27] and Beckwith ${ }^{3}$ is that density fluctuations are influenced by a modification of cosmological density $\rho$ in the Friedmann equations by the proportionality factor given by Bashinsky [27], $\left[1-5 \cdot\left(\rho_{\text {neutrino }} / \rho\right)+\vartheta\left(\left[\rho_{\text {neutrino }} / \rho\right]^{2}\right)\right]$. This proportionality factor for $\rho$ as showing up in the Friedmann equations should be taken as an extension of results from Marklund et al. [7], due to graviton-neutrino interactions as proposed by Marklund et al. [7], where neutrinos interact with plasmons and plasmons interact with gravitons. Thereby implying neutrino-graviton interactions Also, graviton wavelengths have the same order of magnitude of neutrinos. Note, from Valev [28],

$$
\begin{aligned}
& \left.m_{\text {graviton }}\right|_{\text {RELATIVISTIC }}<4.4 \times 10^{-22} h^{-1} \mathrm{eV} / \mathrm{c}^{2} \\
& \Leftrightarrow \lambda_{\text {graviton }} \equiv \frac{\hbar}{m_{\text {graviton }} \cdot c}<2.8 \times 10^{-8} \text { meters }
\end{aligned}
$$

Extending M. Marklund et al. [7] and Valev [28], some gravitons may become $\operatorname{larger}^{14}$, i.e.

$$
\lambda_{\text {graviton }} \equiv \frac{\hbar}{m_{\text {graviton }} \cdot c}<10^{4} \text { meters or larger }
$$

A way to accommodate this wave length as to an instanton-anti instanton packaging of gravitons, was to start with an analogy between Giovannini, [29] from a least action version of the Einstein-Hilbert action for "quadratic" theories of gravity involving Euler-Gauss-Bonnet. Then Giovannini’s [28] Equation (6) corresponds to

$$
\phi=\tilde{v}+\arctan \left((b w)^{v}\right)
$$

Givannini [28] represents of Equation (26a) as a kink, and makes references to an anti-kink solution, in Figure 1 in Givannini [28]. Furthermore the similarity between Equation (26a) and $\phi_{+}(z, \tau)=4 \cdot \arctan \left(\exp \left\{\frac{z+\beta \cdot \tau}{\sqrt{1-\beta^{2}}}\right\}\right)$ in Beckwith’s [24] [25] treatment with regards to density wave physics instantons is obvious. If $\arctan \left((b w)^{v}\right)$ is part of representing a graviton as a kink-anti-kink combination, arising from a 5 dimensional line element, [28]

$$
\mathrm{d} S^{2}=a(w) \cdot\left[\eta_{u v} \mathrm{~d} x^{u} \mathrm{~d} x^{v}-\mathrm{d} w^{2}\right]
$$

The end result of this would be to have an instaton-anti instanton structure as to emergence of a massive graviton if noting, that there is the possibility of using t'Hoofts [17] [22] [23] classical embedding of "deterministic quantum mechanics" as a way to embed a nearly four dimensional graviton as having almost zero mass, in a larger non linear theory.

\section{How DM Would Be Influenced by Gravitons}

The interrelationship of structure of the profile of a DM cluster, with any perturbations DM density profile [29]

$$
\delta \equiv-\left[\frac{3}{2} \cdot \Omega_{m} \cdot H^{2}\right]^{-1} \cdot \nabla^{2} \Phi
$$

As told to the author by Sabino Matarre [29], in July, 2009, in Como Italy, the gravitational potential has, perturbatively speaking an additional term $f_{N L}$ added to variations in the gravitational potential term which Matarre [29] gave as 


$$
\Phi \equiv \Phi_{L}+f_{N L} \cdot\left[\Phi_{L}^{2}-\left\langle\Phi_{L}^{2}\right\rangle\right]+g_{N L} \cdot \Phi_{L}^{3}
$$

It is suggested that the function $f_{N L}$ is largely due to entropy variations, some of which occurred during relic GW/graviton production. Here the expression $f_{N L}=$ variations from gaussianity. Furthermore, $\Phi_{L}$ is a linear Gaussian potential, and the overall gravitational potential is altered by inputs from $f_{N L}$. Note that neutrinos flavor physics oscillations are not very important in terms of $f_{N L}$, as specified in conversations. Beckwith had in September 23, 2009 in Erice with George Raffert [30]. Which leads to emphasizing the role of entropy processes due to graviton-neutrino physics, as $\bar{L} \rightarrow 0$ as written up by Beckwith [31].

\section{1st Part of Massive Graviton Consequences}

The real start to this investigation is to explain how and why the star HE0107-5240 could form with so little lithium in the first place [31]. As stated by Fuller et al. [9] neutrinos could interact with DM potential wells in ways Beckwith thinks could influence deviations from standard galaxy hierarchy formation models which will also have a counter part in deviations in the BBN nucleosynthesis of light elements, by examining the role of temperature fluctuations modeled on Equation (29) below, leading to fluctuations affecting BBN element rarity [31].

$$
(\delta T / T) \cong(1 / 3) \cdot\left[\Phi_{L}+\tilde{f}_{N L} \cdot\left(\Phi_{L}^{2}-\left\langle\Phi_{L}\right\rangle^{2}\right)\right]
$$

While Equation (29) above would have its maximum impact for regions as of about red shift $Z \sim 1.5-2.0$, the impact of Equation (29) would be as of red shifts $Z \sim 1000-1100$, with the corresponding $\tilde{f}_{N L}$ influenced by Bashinsky's [27] neutrino-gravition damping as stated by the coefficient of density fluctuation modified by $\left[1-5 \cdot\left(\rho_{\text {neutrino }} / \rho\right)+\vartheta\left(\left[\rho_{\text {neutrino }} / \rho\right]^{2}\right)\right]$ [27]. Note that $\tilde{f}_{N L}$ would be larger than $f_{N L}$ of Equation (28) and would be dominated by neutrino-graviton interactions, whereas $f_{N L}$ would be dominated by graviton generated entropy, with neutrinos at $Z \sim 2.0$ hitting DM directly. We submit that a graviton with a small rest mass may be more amendable to such interaction with neutrinos, and that in addition Equation (27), Equation (28) and Equation (29) may influence and affect structure formation as seen by the following diagram in Figure 1. Note that this is assuming that early universe interactions which we are talking about eventually play out and reach, with the re acceleration of the universe, as outlined in the 1st half of our document to also be indirectly responsible for the famous "halo merging tree diagram we call Figure 3 below. At or about when

$k \geq k_{\text {equilibrium }} \equiv \tau_{\text {equilibrium }}^{-1} \sim 10^{-2} \mathrm{Mpc}^{-1}$ begins to delineate the neutrino-GW interaction becoming a significant damping impact upon each other, one would be seeing variations from the usual structure formation, as given by the following diagram. [32].

We should keep in mind that the following holds, i.e. for flat space. That one will have Figure 3 in both flat and in curved space. Also note that, M. Marklund, G. Brodin, and P. K. Shukla [7] posted their own version of not only neutrino mass, as given by $m_{v}^{2}=-g_{\alpha \beta} p^{\alpha} p^{\beta}$, where the overall mass is set by Note, here, that the

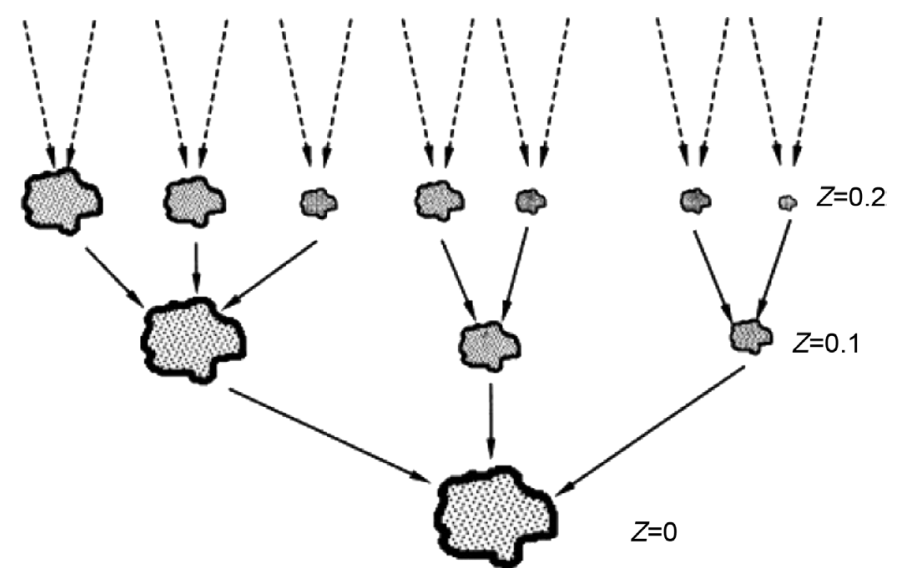

Figure 3. How we obtain "bottom up" development of galactic super structure which duplicates a diagram given in reference [32]. 
potential for where the frequency comes from is, here, is $U=\hbar \cdot \omega_{F}$, and, according to Eberle and Ringwald et al. [33], may have lightest relic neutrino masses of the order of

$$
m_{\text {relic-neutrino }} \propto 0.1 \mathrm{eV} / c^{2}
$$

as opposed to, as given by D. Valev [34]

$$
m_{\text {graviton }} \leq 2 \times 10^{-29} \breve{h}^{-1} \mathrm{eV} / c^{2}
$$

where $\breve{h} \approx 0.65$, is a dimensionless Hubble constant, Very roughly put, for relic early universe conditions, one may be seeing that the neutrino has $10^{28}-10^{29}$ the effective mass than a graviton. Furthermore, for a neutrino we have

$$
\lambda_{k} \approx \frac{h c}{E_{k}}+\frac{h m_{v k}^{2} c^{5}}{2 E_{k}^{3}}
$$

This will tie in directly with a neutrino mass limit we state as [7]

$$
m_{v}^{2}=-g_{\alpha \beta} p^{\alpha} p^{\beta} \equiv\left[\hbar \cdot \sqrt{\left|g_{00}\right| \cdot \omega_{F}^{2}-g_{\alpha \beta} k^{\alpha} k^{\beta}-2 \omega_{F} g_{0 \alpha} k^{\alpha}}\right]^{2} .
$$

If, as if often expected in inflation, space becomes abruptly flat at the onset of inflation, then for a neutrino mass, as the $\bar{L} \underset{\text { approach-to-standard-model-physics }}{\longrightarrow} 0$ will then lead to the following inequality [7] [31]

$$
\begin{aligned}
& m_{v}^{2} \equiv\left[\hbar \cdot \sqrt{\left|g_{00}\right| \cdot \omega_{F}^{2}-g_{\alpha \beta} k^{\alpha} k^{\beta}-2 \omega_{F} g_{0 \alpha} k^{\alpha}}\right]^{2} \\
& \underset{\text { Flat-Space }}{\longrightarrow}\left[\hbar \cdot \sqrt{\left|g_{00}\right| \cdot \omega_{F}^{2}-g_{\alpha \alpha}\left[k^{\alpha}\right]^{2}-2 \omega_{F} g_{00} k^{0}}\right]^{2}>0 \\
& \Leftrightarrow\left|g_{00}\right| \cdot \omega_{F}^{2}>g_{\alpha \alpha}\left[k^{\alpha}\right]^{2}+2 \omega_{F} g_{00} k^{0} \Rightarrow\left|g_{00}\right| \cdot \omega_{F}^{2}>g_{\alpha \alpha}\left[k^{\alpha}\right]^{2}+2 \omega_{F} g_{00} k^{0}
\end{aligned}
$$

Now, how would variation from the above "halo Merging history tree", partly due to the modulation, via entropy, of DM structure formation, due to GW/gravitons affecting DM profile affect the concentration for lithium in stars, and perhaps lead to the famous "lithium problem" being resolved? We are investigating it. But we do think that having a graviton with mass is affecting the particulars of the "halo mixing tree" diagram [32].

\section{2nd Part of Massive Graviton Consequences}

Beckwith [35] has concluded that the only way to give an advantage to higher dimensions as far as cosmology would be to look at if a fifth dimension may present a way of actual information exchange to give the following parameter input from a prior to a present universe, i.e. the fine structure constant, as given by [35]

$$
\tilde{\alpha} \equiv e^{2} / \hbar \cdot c \equiv \frac{e^{2}}{d} \times \frac{\lambda}{h c}
$$

Equation (35) above is in tandem, with examining if the following holds, i.e. for the consistency of physical law, namely from cycle to cycle is there a preservation of Planck's constant? Namely

$$
\hbar(\text { prior-universe })=\hbar \text { (present-universe })
$$

The wave length as may be chosen to do such an information exchange would be part of a graviton as being part of an information counting algorithm as can be put below, namely: Argue that when taking the log, that the $1 / \mathrm{N}$ term drops out. As used by $\mathrm{Ng}[5]$

$$
Z_{N} \sim(1 / N !) \cdot\left(V / \lambda^{3}\right)^{N}
$$

This, according to $\mathrm{Ng}$ [5], leads to entropy of the limiting value of, if $S=\left(\log \left[Z_{N}\right]\right)$ will be modified by having the following done, namely after his use of quantum infinite statistics,

$$
S \approx N \cdot\left(\log \left[V / \lambda^{3}\right]+5 / 2\right) \approx N
$$


Eventually, the author hopes to put on a sound foundation what t'Hooft [22] [23] is doing with respect to t'Hooft [22] [23] deterministic quantum mechanics and equivalence classes embedding quantum particle structures. Doing so will answer the questions Kay $[36]^{29}$ raised about particle creation, and the limitations of the particle concept in curved and flat space, i.e. the global hyperbolic space time which is flat everywhere expect in a localized "bump" of curvature. Furthermore, if we have an initial universe count of gravitons as $S$ (initial) $\sim 10^{10}$ to at most $S$ (initial) $\sim 10^{20}$, we are assuming the existence of one operation per graviton. This one operation per graviton in the early universe may correspond to at least one unit of information per graviton, i.e. one unit of "information" per graviton is correlated directly with one "operation" per graviton. The operation in this case is likely the creation of initial gravitons, in the early universe. This datum needs experimental confirmation and is important to astro physics linkage of DE with DM, in the future. Equation (14) to Equation (17) if confirmed for $Z \sim 0.423$ may prove that higher dimensions are necessary for cosmology.

\section{3rd Massive Graviton Consequences, the Need to Find out the Border of the Introduction of Where Quantum Gravity Emerges from a Prior "Analog" Structure May, If Tied into Questions of Graviton Mass Determine If Multiple Universes Are Possible/Feasible}

Beckwith [37], in his FQXi document outlined a procedure where a graviton with mass may be indicative of the existence of multiple universes co existing. The details of the mapping of that multiple universe picture involve a transition from an analog physics (discrete, i.e. classical world picture) to one where octonian gravity is formed, i.e. a quantum picture as a pre cursor to quantum gravity. The existence of a small mass may mean the extension of quantum physics to a larger embedding/extension of quantum physics. Furthermore, keep in mind that tandem to that step of semi classical embedding of a graviton, that eventually we want to make explicit an idea by, T. Padmanabhan in DICE 2010 [38], as to finding "atoms of space time” permitting a thermodynamic treatment of emergent structure similar to Gibbs treatment of statistical physics. i.e. for finding out if the following is possible, i.e. can an ensemble of gravitons, be used to construct an "atom" of space time congruent with relic GW. That is our ultimate end, as to our research. That would make our inquiry of the nature of gravitons most worthwhile. This idea was presented at DICE 2010, [39] and we would like to refine it in our future research work. This would be in tandem of adapting the Kiefer, Polarski, and Starobinsky [40] presentation of the evolution of relic entropy via the evolution of phase spaces, with $\Gamma / \Gamma_{0}$ being the ratio of "final (future)"/“initial” phase space volume, for $k$ modes of secondary GW background. From "atoms of space time" treatment of early universe space time geometry according to [40]

$$
S(k)=\ln \frac{\Gamma}{\Gamma_{0}}
$$

This lead to the author, Beckwith to derive the following a important for structure formation, note the following about what happens if $g_{*} \geq 120$

$$
\Omega \sim \frac{\left[\frac{\pi^{2}}{30} \cdot g_{*} \cdot\left[1.416 \times 10^{19} \mathrm{GeV}\right]^{4}\right]}{3 \cdot\left[1.2 \times 10^{19} \mathrm{GeV}\right]^{2} H_{\text {initial }}^{2}} \sim \frac{\frac{\pi^{2}}{30} \cdot g_{*} \cdot c^{4}}{3} \cdot \frac{\left[10^{19} \mathrm{GeV}\right]^{2}}{H_{\text {initial }}^{2}} \geq 1
$$

i.e. especially if the degrees of freedom rises above $g_{*} \geq 120$.

Note that $g_{*} \approx 120$ at $T \sim 100 \mathrm{KeV}$ Unless the term for $H_{\text {initial }}$ were absolutely enormous, and if $g_{*} \approx 1000$, then $\Omega \geq 1$ could happen, which would be physically meaningless. The other situation is that there could be situations for which $g_{*}$ would be undefined, especially if $T \leq 1.416 \times 0^{32} \mathrm{~K} \sim 10^{19} \mathrm{GeV}$ were close to an equality. We state here unequivocally that Equation (38) and Equation (39) above are important, and that this has serious experimental import. Having said that, we will next go to what would be a way to determine if Gravitons can have mass (massive Gravitons). i.e. in the conclusions section, we radically extend the consequences if $g_{*} \geq 120$, with a speculation as to what could happen as to dark matter and dark energy contributions, which we think is important to the matter of singularities and their purported connection to a multiverse. But before we 
get to that matter, we will examine the role of partition functions, in terms of background which will lead to several pages later, to $g_{*} \geq 120$ contributions, especially for the regime of values, say of 1100 to 1200 , which we think has to be seriously looked at.

\section{Working with a Partition Function Argument in the Case of a Multiverse}

This section is to determine if gravitons have mass and backs the assertion made earlier that multiverse construction has massive gravitons. Note that this section is directly linked to the first part of this document, as to what was done by the author to extend Kauffman's work [1].

We assume that there are no fewer than $N$ universes undergoing Penrose "infinite expansion" (Penrose, 2006) [41]-[43] contained in a mega universe structure. Furthermore, each of the $N$ universes has black hole evaporation, with the Hawking radiation from decaying black holes. If each of the $N$ universes is defined by a partition function, called $\left\{\Xi_{i}\right\}_{i=N}^{i=1}$, then there exist an information ensemble of mixed minimum information correlated as about $10^{7}-10^{8}$ bits of information per partition function in the set $\left.\left\{\Xi_{i}\right\}_{i=N}^{i=1}\right|_{\text {before }}$, so minimum information is conserved between a set of partition functions per universe [44]

$$
\left.\left.\left\{\Xi_{i}\right\}_{i=N}^{i=1}\right|_{\text {before }} \equiv\left\{\Xi_{i}\right\}_{i=N}^{i \equiv 1}\right|_{\text {after }}
$$

However, there is non-uniqueness of information put into each partition function $\left\{\Xi_{i}\right\}_{i=N}^{i=1}$. Furthermore Hawking radiation from the black holes is collated via a strange attractor collection in the mega universe structure to form a new big bang for each of the $N$ universes represented by $\left\{\Xi_{i}\right\}_{i=N}^{i=1}$. Verification of this mega structure compression and expansion of information with a non-uniqueness of information placed in each of the $\mathrm{N}$ universes favors ergodic mixing treatments of initial values for each of $N$ universes expanding from a singularity beginning. The $n_{f}$ value, will be using $S_{\text {entropy }} \sim n_{f}$. How to tie in this energy expression, as in Equation (40) will be to look at the formation of a nontrivial gravitational measure as a new big bang for each of the $N$ universes as by $n\left(E_{i}\right)$. the density of states at a given energy $E_{i}$ for a partition function. (Poplawski, 2011) [45]

$$
\left\{\Xi_{i}\right\}_{i=1}^{i \equiv N} \propto\left\{\int_{0}^{\infty} \mathrm{d} E_{i} \cdot n\left(E_{i}\right) \cdot \mathrm{e}^{-E_{i}}\right\}_{i=1}^{i \equiv N} .
$$

Each of $E_{i}$ identified with Equation (41) above, are with the iteration for $N$ universes (Penrose, 2006) [41]-[44] Then the following holds, namely [44]

Claim 1, [44]

$$
\left.\left.\frac{1}{N} \cdot \sum_{j=1}^{N} \Xi_{j}\right|_{j \text {-before-nucleation-regime }} \stackrel{ }{\text { vacuum-nucleation-tranfer }} \Xi_{i}\right|_{i \text {-fixed-after-nucleation-regime }}
$$

For $N$ number of universes, with each $\left.\Xi_{j}\right|_{j \text {-before-nucleation-regime }}$ for $j=1$ to $N$ being the partition function of each universe just before the blend into the RHS of Equation (42) above for our present universe. Also, each of the independent universes given by $\left.\Xi_{j}\right|_{j \text {-before-nucleation-regime }}$ are constructed by the absorption of one to ten million black holes taking in energy. i.e. (Penrose, 2006) [41]-[44]. Furthermore, the main point is similar to what was done in [18] in terms of general ergodic mixing

Claim 2 [44]

$$
\left.\left.\Xi_{j}\right|_{j \text {-before-nucleation-regime }} \approx \sum_{k=1}^{\text {Max }} \tilde{\Xi}_{k}\right|_{\text {black-holes-jth-universe }}
$$

Claim 3 The idea here is to use what is known as CCC cosmology [41]-[44].

First. Have a big bang (initial expansion) for the universe. After red shift $z=10$, a billion years ago, SMBH formation starts. Matter-energy is vacuumed up by the SMBHs, which at a much later date than today (present era) gather up all the matter-energy of the universe and recycles it in a cyclic conformal translation, as follows, namely 
$E=8 \pi \cdot T+\Lambda \cdot g$

$E=$ source for gravitational field

$T=$ mass energy density

$g=$ gravitational metric

$\Lambda$ = vacuum energy, rescaled as follows

$$
\Lambda=c_{1} \cdot[\text { Temp }]^{\beta}
$$

$c_{1}$ is a constant. Then the main methodology in the Penrose proposal has been in Equation (45) evaluating a change in the metric $g_{a b}$ by a conformal mapping $\hat{\Omega}$ [43] [44] to

$$
\hat{g}_{a b}=\hat{\Omega}^{2} g_{a b}
$$

Penrose's suggestion has been to utilize the following [43]

$$
\hat{\Omega} \underset{c c c}{\longrightarrow} \hat{\Omega}^{-1}
$$

Infall into cosmic black hopes has been the main mechanism which the author asserts would be useful for the recycling apparent in Equation (47) above with the caveat that $\hbar$ is kept constant from cycle to cycle as represented by a restatement of Equation (35a) as in the multiverse as

$$
\hbar_{\text {old-cosmology-cycle }}=\hbar_{\text {present-cosmology-cycle }}
$$

Equation (47) is to be generalized, as given by a weighing averaging as given by Equation (42) where the averaging is collated over perhaps thousands of universes, call that number $N$, with an ergodic mixing of all these universes, with the ergodic mixing represented by Equation (42) to generalize Equation (47) from cycle to cycle.

\section{Why This Just Outlined Multiverse Averaging Procedure Implies a Graviton with Mass. Also Why a Single Repeating Universe Has No Massive Gravitons}

In this chapter, we are looking at a generalization of Kolb and Turner's [46] gravitational radiation result which is given as

$$
\frac{k}{\rho_{\text {Horizon }}} \cdot \frac{\mathrm{d} \rho_{\text {graviton }}}{\mathrm{d} k} \sim \frac{4}{3 \pi} \cdot\left(\frac{H}{m_{\text {Planck }}}\right)^{2}
$$

In the immediate aftermath of inflation, and just before inflation, we generalize $\frac{4}{3 \pi} \cdot\left(\frac{H}{m_{\text {Planck }}}\right)^{2}$ as a constant, as well as approximate $\rho_{\text {Horizon }}$ as a constant, with also putting in [34]

$$
\lambda_{\text {graviton }} \equiv \frac{2 \pi}{k}=\frac{\hbar}{c \cdot m_{\text {graviton }}}
$$

Then we have that if "Before" is just before the formation of the present universe, and "Final" is just after the formation of the present universe

$$
\log \frac{\left.\rho_{\text {graviton }}\right|_{\text {Before }}}{\left.\rho_{\text {graviton }}\right|_{\text {Final }}}=\frac{4 \rho_{\text {Horizon }}}{3 \pi} \cdot\left(\frac{H}{m_{\text {Planck }}}\right)^{2} \cdot\left[\frac{1}{\left.m_{\text {graviton }}\right|_{\text {Before }}}-\frac{1}{\left.m_{\text {graviton }}\right|_{\text {Final }}}\right]
$$

Claim 4, in the case of a single repeating Universe, the RHS of (51) is zero, leading to $\left.m_{\text {graviton }}\right|_{\text {Before }}=\left.m_{\text {graviton }}\right|_{\text {Final }}$ implying that the mass of a graviton in a single repeating universe is zero.

Proof: We will use the following value of the net energy, i.e. if $\beta=\frac{1}{k_{\mathrm{Boltzman}} \cdot T(\text { temp })}$

$$
\bar{E}_{N(\text { fixed })}=-\frac{\partial}{\partial \beta}\left\{\Xi_{i=N(\text { fixed })}\right\} \propto-\frac{\partial}{\partial \beta}\left\{\int_{0}^{\infty} \mathrm{d} E_{i} \cdot n\left(E_{i}\right) \cdot \mathrm{e}^{-E_{i}}\right\}_{i=N(\text { fixed })}
$$


Now define an average gravitational energy as given by having a single universe, denoted by $N$ (fixed), i.e. one universe out of $N$ of them [maybe infinite] given as

$$
\left.\bar{\rho}_{\text {graviton }}\right|_{N(\text { fixed })}=\frac{\left.\bar{E}_{\text {graviton }}\right|_{N(\text { fixed })}}{\left.V(\text { volume })\right|_{N(\text { fixed })} \cdot c^{2}}
$$

This is the single universe, repeated, i.e. in this case, we assume that the Volume, per single repeating universe, is the same for a regime of the BB immediately before and after the cosmic explosion. Hence, we have that.

In terms of equipartition function definitions, and to rewrite Equation (52) as in the case of a multiverse, i.e. one out of $N$ "universes"

$$
\bar{E}_{N \text { (fixed) }} \sim \frac{1}{N(\text { full-range })} \cdot \frac{\sum_{r=1}^{\infty}\left[\exp \left(-\beta \cdot \sum_{j=1}^{N} E_{j}\right)\right]_{r} \cdot\left[-\beta \cdot \sum_{j=1}^{N} E_{j}\right]_{r}}{\sum_{r=1}^{\infty}\left[\exp \left(-\beta \cdot \sum_{j=1}^{N} E_{j}\right)\right]_{r}}
$$

It so happens, then that there are $r$ "states" per universe, and an infinite number of them. Then the average graviton radiation density would be, for $r=1$ to infinite number of energy states per $N$ th universe, with the label $N$ (full-range) being the number of universe domains in a multiverse.

$$
\left.\bar{\rho}_{\text {graviton }}\right|_{N \text { (fixed) }}=\frac{1}{N(\text { full-range })} \cdot \frac{\sum_{r=1}^{\infty}\left[\exp \left(-\beta \cdot \sum_{j=1}^{N} E_{j}\right)\right]_{r} \cdot\left[-\beta \cdot \sum_{j=1}^{N} E_{j}\right]_{r}}{\left.V(\text { volume })\right|_{N(\text { fixed })} \cdot c^{2} \sum_{r=1}^{\infty}\left[\exp \left(-\beta \cdot \sum_{j=1}^{N} E_{j}\right)\right]_{r}}
$$

In terms of the averaging procedure of Equation (42), we then have the initial and final states for the multiverse as

$$
\begin{gathered}
\frac{\left.\left.\bar{\rho}_{\text {graviton }}\right|_{N(\text { fixed })}\right|_{\text {before-BB }} \neq\left.\left.\bar{\rho}_{\text {graviton }}\right|_{N(\text { fixed })}\right|_{\text {after-BB }}}{N(\text { full-range })} \cdot \frac{\sum_{r=1}^{\infty}\left[\exp \left(-\beta \cdot \sum_{j=1}^{N} E_{j}\right)\right] \cdot\left[-\beta \cdot \sum_{j=1}^{N} E_{j}\right]_{r}}{\left.\left.V(\text { volume })\right|_{N(\text { fixed })} \cdot c^{2} \sum_{r=1}^{\infty}\left[\exp \left(-\beta \cdot \sum_{j=1}^{N} E_{j}\right)\right]\right]\left._{r}\right|_{\text {Just-Before-present-universe }}} \\
\left.\frac{\sum_{r=1}^{\infty}\left[\exp \left(-\beta \cdot E_{N(\text { fixed })}\right)\right]_{r} \cdot\left[-\beta \cdot E_{N(\text { fixed })}\right]_{r}}{\left.V(\text { volume })\right|_{N(\text { fixed })} \cdot c^{2} \sum_{r=1}^{\infty}\left[\exp \left(-\beta \cdot E_{N(\text { fixed })}\right)\right]_{r}}\right|_{N(\text { fixed }) \text {-start-inf }}
\end{gathered}
$$

This would be due to the behavior of $\left[-\beta \cdot \sum_{j=1}^{N} E_{j}\right]_{r}$ before the big bang, which will lead to

$$
=\frac{\left.\left.\bar{\rho}_{\text {graviton }}\right|_{N(\text { fixed })}\right|_{\text {before-BB }}}{N(\text { full-range })} \cdot \frac{\sum_{r=1}^{\infty}\left[\exp \left(-\beta \cdot \sum_{j=1}^{N} E_{j}\right)\right]_{r} \cdot\left[-\beta \cdot \sum_{j=1}^{N} E_{j}\right]_{r}}{\left.V(\text { volume })\right|_{N(\text { fixed })} \cdot c^{2} \sum_{r=1}^{\infty}\left[\left.\left.\exp \left(-\beta \cdot \sum_{j=1}^{N} E_{j}\right)\right|_{r}\right|_{\text {Just-Before-present-universe }}\right.}
$$


Which should be compared to

$$
\left.\left.\bar{\rho}_{\text {graviton }}\right|_{N(\text { fixed })}\right|_{\text {after-BB }}=\left.\frac{\sum_{r=1}^{\infty}\left[\exp \left(-\beta \cdot E_{N(\text { fixed })}\right)\right]_{r} \cdot\left[-\beta \cdot E_{N(\text { fixed })}\right]_{r}}{\left.V(\text { volume })\right|_{N(\text { fixed })} \cdot c^{2} \sum_{r=1}^{\infty}\left[\exp \left(-\beta \cdot E_{N(\text { fixed })}\right)\right]_{r}}\right|_{N(\text { fixed)-start-inf }}
$$

Equation (58) and Equation (59) above are not the same value, hence the results given in Equation (56). Hence the masses of the gravitons would not be the same by Equation (51).

Note that Feynman and Hibbs [47] have a different way of writing a net energy as can be written using $E_{i}$ as the total energy of the ith universe, and $E_{r}=$ energy of the $r$ th sub domain of the ith universe i.e. two different energy expressions.

$$
\left.\left.\Xi_{i}\right|_{i \text {-fixed,one-universe }} \propto\left\{\int_{0}^{\infty} \mathrm{d} E_{i} \cdot n\left(E_{i}\right) \cdot \mathrm{e}^{-E_{i}}\right\}\right|_{\text {i-fixed,one-universe }} \sim \sum_{r} \exp \left[-\beta E_{r}\right]
$$

Then, using Feynman and Hibbs [47], the net energy can be written as

$$
\bar{E} \equiv \frac{\sum_{r=1}^{\infty} E_{r} \cdot \exp \left(-\beta \cdot E_{r}\right)}{\left.\Xi_{i}\right|_{i-\text { fixed,one-universe }}} \propto \frac{\left.\sum_{r=1}^{\infty} E_{r} \cdot \exp \left(-\beta \cdot E_{r}\right)\right|_{r \text {-subdomains,i-fixed,one-univerese }}}{\left.\left\{\int_{0}^{\infty} \mathrm{d} E_{i} \cdot n\left(E_{i}\right) \cdot \mathrm{e}^{-E_{i}}\right\}\right|_{\text {i-fixed,one-universe }}}
$$

The results as outlined above are, again then, more obvious.

\section{Conclusions and Further Tests as Far as Upper Bounds to a Graviton Mass. with Consequences}

First of all, the contributions of Gravitons to reacceleration of the universe are outlined as a consequence of massive gravitons. In addition, the graviton mass of a non zero value is central to the process of entropy generation which leads to our next comment which is a further research project in its own right. For what it is worth, we will address an extension of an entropy versus graviton production linkage implied in the first linkage. This entropy versus gravition linkage, as seen below, will imply a non zero initial radius for the universe. Before that is brought up, we should consider entropy generation with an initial cosmological "constant" (vacuum energy) at the start of inflation.

\subsection{Difficulty in Visualizing What $g *$ Is in the Initial Phases of Inflation}

Secondly, we look for a way to link initial energy states, which may be pertinent to entropy, in a way which permits an increase in entropy from about $10^{10}$ at the start of the big bang to about $10^{100}$ today.

One such way to conflate entropy with an initial cosmological constant may be of some help, i.e. if

$\left.V_{4}\right|_{\text {Threshold-volume-for-quantum-effects }} \sim\left(10^{-4} \mathrm{~cm}\right)^{3}$ or smaller, i.e. in between the threshold value, and the cube of Planck length, We change the cosmological constant, as given by Padmabhan, with $\Lambda_{\max }$ defined via Equation (62), in the referenced equation below as given by Padmanabhan [17]

$$
\frac{\Lambda_{\max } V_{4}}{8 \cdot \pi \cdot G} \sim T^{00} V_{4} \equiv \rho \cdot V_{4}=E_{\text {total }}
$$

Then make the following identification of total energy with entropy via looking at $\Lambda_{\max }$ models, i.e. consider Park's model of a cosmological “constant” parameter scaled via background temperature [48]

$$
\Lambda_{\max } \sim c_{2} \cdot T^{\tilde{\beta}}
$$

A linkage between energy and entropy may be seen in the following construction, namely looking at what Kolb puts in [46], i.e. 


$$
\rho=\rho_{\text {radiation }}=(3 / 4) \cdot\left[\frac{45}{2 \pi^{2} g_{*}}\right]^{1 / 3} \cdot S^{4 / 3} \cdot r^{-4}
$$

Here, we in the following Equation (65) derive an explicit relationship between maximum initial cosmological vacuum energy and the initial entropy, of $10^{10}$, at the initial beginning of cosmological expansion

$$
\left[\left[\frac{\Lambda_{\max } r^{4}}{8 \pi G}\right] \cdot(4 / 3) \cdot\left[\frac{2 \pi^{2} g_{*}}{45}\right]^{1 / 3}\right]^{3 / 4} \sim S_{\text {initial }}
$$

Note that in the case that quantum effects become highly significant and that the contribution as given by

$\left.V_{4}\right|_{\text {Threshold-volume-for-quantum-effects }} \sim\left(10^{-4} \mathrm{~cm}\right)^{3}$ and potentially much smaller, as in the threshold of Plancks length, going down to possibly as low as $4.22419 \times 10^{-105} \mathrm{~m}^{3}=4.22419 \times 10^{-96} \mathrm{~cm}^{3}$ leads us to conclude that even with very high temperatures, as an input into the initial entropy, $S_{\text {initial }} \approx 10^{10}$ is very reasonable. Note that even if we have an initial non Zero entropy, Kolb and Turner still have the initial degrees of freedom $g_{*}$ as with an upper bound of 120, in contravention of exotic beyond the standard models with significantly higher initial degrees of freedom, whereas the author, in conversation with H. De La Vega, in 2009 [49] indicates that even the exotic theories of $g_{*}$ have an upper limit of about 1200, and that it is difficult to visualize what $g_{*}$ is in the initial phases of inflation. De La Vega stated in Como Italy, that he, as a conservative cosmologist, viewed defining $g_{*}$ in the initial phases of inflation as impossible [49]. If the DM and DE contributions to $g_{*}$ are allowed, then this supposition as given by [49] is then drawn into question.

One should not assume that the issue (does a non zero initial radii of the universe exist) is of decisive importance for the following, i.e. determining conditions for either supporting or denying the existence of non zero initial entropy, whereas we claim that non zero entropy is necessary in information exchange. How we break out of the alleged circular reasoning is to go back again to the datum of (48), namely we assert non zero initial entropy, to exchange information, in order to seek having the following hold from cycle to cycle.

The following will be what is to be worked upon, namely for now assuming that we can break down the degrees of freedom question as follows,

$$
\left.g_{*}\right|_{\text {initial }} \cong\left(\left.g_{*}\right|_{\text {Baryons-initial }} \sim 110-120\right)+\left(\left.g_{*}\right|_{(\text {DM-DE)-initial }} \sim 1000\right) \equiv 1100-1200
$$

The figure for the first entry is from Kolb and Turner, and what we assume we have to investigate is the bona fides of looking at what happens due to

$$
\left.g_{*}\right|_{(\mathrm{DM}-\mathrm{DE}) \text {-initial }}=-\left.g_{*}\right|_{\text {Baryons-initial }}+\left(\frac{45}{2 \pi^{2}}\right) \cdot\left(\frac{3}{4}\right)^{3} \cdot \frac{\left(S_{\text {initial }}\right)^{4}}{\left[\frac{\Lambda_{\text {max }} \cdot r^{4}}{8 \pi G}\right]^{3}}
$$

The details of this derivation would assume that there would be a multiverse, that secondly there would be an initial entropy, and most likely, that there would be a non zero initial radii for the start of our present universe. Finally, this is a phenomenological prediction which should be tested, namely, experimental tests which may permit upper bound tests as to the mass of a graviton. The following section makes references to interstellar tests which give upper bound values, which may indicate how the approximation by [1] may be utilized.

\subsection{How the CMBR Permits, via Maximum Frequency, and Maximum Wave Amplitude Values, an Upper Bound Value for Massive Graviton Mass $m_{g}$}

Camp and Cornish (2004) [50] use the typical transverse gravitational gauge $h_{i j}$ with a typically traceless value summed as $0+h_{+}-h_{+}+0$ and off diagonal elements of $h_{x}$ on each side of the diagnonal to mix with a value of

$$
h_{i j} \equiv \frac{G_{N}}{c^{4}} \cdot \frac{2}{r} \cdot\left[\frac{\mathrm{d}^{2}}{\mathrm{~d} t^{2}} Q_{i j}\right]_{\text {retarded }}^{T T}
$$

This assumes $r$ is the distance to the source of gravitational radiation, with the retarded designation on Equa- 
tion (68) denoting $\frac{\mathrm{d}}{\mathrm{d} t}$ replaced by a retarded time derivative $\frac{\mathrm{d}}{\mathrm{d}[t-(r / c)]}$, while TT means taking the transverse projections and substracting the trace. Here, we call the quadrupole moment, with $\rho(t, x)$ a density measurement. Now, the following value of the $Q_{i j}$ as given gives a luminosity function $L$, where $R$ is the "characteristic size" of a gravitational wave source. Note that if $M$ is the mass of the gravitating system [50].

$$
\begin{gathered}
Q_{i j}=\int \mathrm{d}^{3} x\left[x_{i} x_{j}-\frac{1}{3} \cdot \delta_{i j} \cdot x^{2}\right] \cdot \rho(t, x) \\
L \approx \frac{1}{5} \cdot \frac{G_{N}}{c^{5}} \cdot \frac{\mathrm{d}^{3} Q_{i j}}{\mathrm{~d} t^{3}} \cdot \frac{\mathrm{d}^{3} Q^{i j}}{\mathrm{~d} t^{3}} \cong \frac{\pi \cdot c^{5}}{G_{N}} \cdot\left(\frac{G_{N} M}{R \cdot c^{2}}\right)^{2}
\end{gathered}
$$

After certain considerations reported by Camp and Cornish (2004), one can recover a net GW amplitude

$$
h \sim 2 \cdot\left[\frac{G_{N} \cdot M}{R \cdot c^{2}}\right] \cdot\left[\frac{G_{N} \cdot M}{r \cdot c^{2}}\right]
$$

This last equation requires that $R>R_{G}=\frac{G_{N} M}{c^{2}} \equiv$ gravitational radius of a system, with a black hole resulting if one sets $R<R_{G}=\frac{G_{N} M}{c^{2}}$. Note that when $R \sim R_{G}=\frac{G_{N} M}{c^{2}}$ we are at an indeterminate boundary where one may pick our system as having black hole properties.

Now for stars, Camp and Cornish (2004) [50] give us that

$$
\begin{aligned}
& h \approx 10^{-21} \cdot\left[\frac{15 \mathrm{Mpc}}{r}\right] \cdot\left[\frac{M}{2.8 M_{\text {solar-mass }}}\right]^{2} \cdot\left(\frac{90 \mathrm{~km}}{R}\right) \\
& f \equiv \text { frequency } \approx \sqrt{\frac{M}{2.8 M_{\text {solar-mass }}}} \cdot \sqrt{\frac{90 \mathrm{~km}}{R}} \cdot 100 \mathrm{~Hz}
\end{aligned}
$$

As well as a mean time $\tau_{G W}$ for half of gravitational wave potential energy to be radiated away as

$$
\tau_{G W} \approx \frac{R}{2 \pi \cdot c} \cdot\left[\frac{G_{N} M}{R \cdot c^{2}}\right]^{-3} \sim\left(\frac{R}{90 \mathrm{~km}}\right)^{4} \cdot\left[\frac{2.8 M_{\text {solar-mass }}}{M}\right]^{3} \cdot\left(\frac{1}{2} \cdot \mathrm{sec}\right)
$$

The assumption we make is that if we model $R \sim R_{G}=\frac{G_{N} M}{c^{2}}$, for a sufficiently well posed net mass $M$ that the star formulas roughly hold for early universe conditions, provided that we can have a temperature $T$ for which we can use the approximation $\approx \sqrt{\frac{M}{2.8 M_{\text {solar-mass }}}} \cdot \sqrt{\frac{90 \mathrm{~km}}{R}} \cdot 100 \mathrm{~Hz}$ that we also have $\left[\frac{T}{\mathrm{TeV}}\right] \sim 10^{13}$ or higher, so that at a minimum we recover Grishchuck's [51] value of

$$
\begin{aligned}
f_{\text {Peak }} & \approx\left(10^{-3} \mathrm{~Hz}\right) \cdot\left[\frac{T}{\mathrm{TeV}}\right] \sim 10^{10} \mathrm{~Hz} \\
& \approx \sqrt{\frac{M}{M_{\text {solar-mass }}}} \cdot \sqrt{\frac{90 \mathrm{~km}}{R}}
\end{aligned}
$$

Equation (75) places, for a specified value of $R$, which can be done experimentally, an upper bound as far as what a mass $M$ would be. Can this be exploited to answer the question whether or not there is a minimum value for the Graviton mass? The key to the following discussion will be that

$$
\sqrt{\frac{M}{2.8 M_{\text {solar-mass }}}} \cdot \sqrt{\frac{90 \mathrm{~km}}{R}} \approx 10^{8} \text { or larger }
$$




\subsection{Inter Relationship between Graviton Mass $m_{g}$ and the Problem of a Sufficient Number of Bits of $\hbar$ from a Prior Multiverse Contribution to the Present Universe, to Preserve Continuity between Fundamental Constants Namely Planck's Constant}

P. Tinyakov (2006) [52] gives that there is, with regards to the halo of sub structures in the local Milky Way galaxy an amplitude factor for gravitational waves of

$$
\left\langle h_{i j}\right\rangle \sim 10^{-10} \cdot\left[\frac{2 \times 10^{-4} \mathrm{~Hz}}{m_{\text {graviton }}}\right]
$$

If we use LISA values for the Pulsar Gravitational wave frequencies, this may mean that the massive graviton is ruled out. On the other hand $\sqrt{\frac{M}{2.8 M_{\text {solar-mass }}}} \cdot \sqrt{\frac{90 \mathrm{~km}}{R}} \approx 10^{8}$ as proportional to the initial entropy leads to looking at, if

$$
\left\langle h_{i j}\right\rangle \sim h \sim 10^{-5} \cdot\left[\frac{15 \mathrm{Mpc}}{r}\right]^{1 / 2} \cdot\left[\frac{M}{2.8 \cdot M_{\text {solar-mass }}}\right]^{1 / 2} \approx 10^{-30}
$$

If the radius is of the order of $r \geq 10$ billion light-years $\sim 4300 \mathrm{Mpc}$ or much greater, then we have, as an example

$$
\begin{gathered}
\left\langle h_{i j}\right\rangle \sim 10^{-10} \cdot\left[\frac{2 \times 10^{-4} \mathrm{~Hz}}{m_{\text {graviton }}}\right] \approx 5.9 \times 10^{-7} \cdot\left[\frac{M}{2.8 \cdot M_{\text {solar-mass }}}\right]^{1 / 2} \\
{\left[\frac{10^{-7} \mathrm{~Hz}}{m_{\text {graviton }}}\right] \approx\left[\frac{5.9}{\sqrt{5.6}}\right] \cdot \sqrt{\frac{M}{M_{\text {solar-mass }}}}}
\end{gathered}
$$

Equation (71) is in units where $\hbar=c=1$.

If $10^{-60}$ grams per graviton, and 1 electron volt is in rest mass, so $10^{1.6 \times 10^{-33}}$ grams $\Rightarrow$ gram $=6.25 \times 10^{32} \mathrm{eV}$. Then

$$
\left[\frac{10^{-7} \mathrm{~Hz}}{m_{\text {graviton }}}\right] \equiv\left[\frac{10^{-7} \mathrm{~Hz} \cdot\left[6.582 \times 10^{-15} \mathrm{eV} \cdot \mathrm{s}\right]}{\left[10^{-60} \text { grams } \equiv 6.25 \times 10^{-28} \mathrm{eV}\right] \cdot\left[2.99 \times 10^{9} \mathrm{~meter} / \mathrm{sec}\right]^{2}}\right] \sim \frac{10^{-22}}{10^{-9}} \sim 10^{-13}
$$

Then, there exist

$$
M \sim 10^{-26} M_{\text {solar-mass }} \approx 1.99 \times 10^{33-26} \equiv 1.99 \times 10^{7} \text { grams . }
$$

Conceivably this mass $M$ would be transferred from a prior multiverse to a present universe, and may have been enough to preserve the value of Planck's constant in the sense of what is represented in (48), as given above. This has much to do with the assumptions as given in [52]-[54] and should be experimentally tested as soon as possible. Particularly the value of Equation (81) is a counterpart to the values calculated in [54], while different in absolute magnitude, the same procedure is in common between Equation (82) and reference [54].

Of special note, is [55], namely that gravitational waves have been discovered so that one can say with confidence, that LIGO.

Observed a transient gravitational-wave signal. The signal sweeps upwards in frequency from 35 to $250 \mathrm{~Hz}$ with a peak gravitational-wave strain of $1.0 \times 10^{\wedge} \_21$. It matches the waveform predicted by general relativity.

Hence, we have a pretty good idea that at least the outward forms of General relativity have been experimentally vetted. This needs to be contrasted with [29], in which if there are Gaussianity or non Gaussianity issues to contend with, as far as gravitational waves, that the data of [54] be vetted. In addition, the experimentally verified details as of reference [56] concerning two black holes generating Gravitational waves have crucial experimental detail. The reference [56] has the following quote 
We constrain the graviton Compton wavelength in a hypothetical theory of gravity in which the graviton is massive and place a $90 \%$-confidence lower bound of $10^{\wedge} 13 \mathrm{~km}$. Within our statistical uncertainties, we find no evidence for violations of general relativity in the genuinely strong-field regime of gravity.

i.e. General relativity appears to hold up well, but in terms of configuring admissible values of a massive graviton, as alluded to in this document, it would be appropriate to review data as to the presumed Compton wavelength of a "massive graviton" and to insure that it is commensurate with Section 13.3 above. i.e. we view that it is, but that in the future we should make the great refinements outlined as given in Section 13.3 which should be adhered to, once the procedures of [56] are refined via additional experimentation.

Finally, and not least is, that the ultimate goal should be to determine the utility of not only [56] but of [57], i.e. to determine if scalar-tensor gravity, which would be commensurate with 3, instead of 2 polarization states for gravitation, or classical General relativity is favored by the data. Correct review of [55] and [56] plus refinements of Section 13.3 will hopefully allow researchers to determine this, and it would be through utilization of

accurate angular and frequency dependent response functions of interferometers for GWs arising from various Theories of Gravity, i.e. General Relativity and Extended Theories of Gravity, will be the definitive test for General Relativity.

The good news is that we are through [55] and [56] learning enough so as to make this determination, and it has to do with refinement of enough information to look at frequency response functions, which was a particular focal point of [55] as to their very careful LIGO work.

In doing all of this it is useful to keep in mind that [55] to [57] plus review of Section 13.3 above will permit the following, namely as was stressed in an interaction the author had with the editors of this journal, that

the realization of gravitational wave astronomy will be important for discriminating among general relativity and other gravity theories

The above Section 13.3 and references [55] to [57], if considerably refined, will lead to such a goal being accomplished. The author looks forward to this happy occurrence once it commences with the birth of gravitational wave astronomy.

Finally what we will be doing through reference [58] is to take the analogy of instaton-anti instaton nucleation given in Figure 2 above a step further. What we obtain is possibly a way to link SO (4) theory and symmetry breaking to an alternative to the usual Higgs boson formation of mass, assuming that the Graviton has a slight mass. This requires serious analytical work and will be followed up in future publications. It likely entails further developments linking reference [58] to reference [59].

\section{Acknowledgements}

This work is supported in part by National Nature Science Foundation of China grant No. 11375279.

\section{References}

[1] Kauffman, S. (2012) A Self Gravitational Upper Bound on Localized Energy Including that of Virtual Particles and Quantum Fields, which Yield a Passable Dark Energy Density Estimate. http://arxiv.org/abs/1212.0426

[2] Beckwith, A. (2011) What Violations of the Null Energy Condition Tell Us about Information Exchange between Prior to Present Universes? How to Obtain Spectral Index Confirmation? http://vixra.org/abs/1102.0039

[3] Dye, H.A. (1965) On the Ergodic Mixing Theorem. Transactions of the American Mathematical Society, 118, $123-130$. http://dx.doi.org/10.1090/S0002-9947-1965-0174705-8

[4] Gao, C. (2012) A Model of Nonsingular Universe. Entropy, 14, 1296-1305. http://dx.doi.org/10.3390/e14071296

[5] Ng, Y.J. (2008) Spacetime Foam: From Entropy and Holography to Infinite Statistics and Nonlocality. Entropy, 10, 441-461. http://dx.doi.org/10.3390/e10040441

[6] Maggiore, M. (2008) Gravitational Waves, Volume 1: Theory and Experiment. Oxford University Press, Oxford.

[7] Marklund, M., Brodin, G. and Shukla, P. (1999) Interaction of Neutrinos and Gravitons with Plasmas in the Universe. Physica Scripta, T82, 130-132.http://dx.doi.org/10.1238/Physica.Topical.082a00130

[8] Battisti, M.V. (2009) Cosmological Bounce from a Deformed Heisenberg Algebra. Physical Review D, 79, Article ID: 
083506. http://dx.doi.org/10.1103/PhysRevD.79.083506

[9] Fuller, G. and Kishimoto, C. (2009) Quantum Coherence of Relic Neutrinos. Physical Review Letters, 102, Article ID: 201303. http://dx.doi.org/10.1103/PhysRevLett.102.201303

[10] Maartens, R. (2005) Brane World Cosmology. In: Papantronopoulos, Ed., The Physics of the Early Universe, Springer, Heidelberg, 213-247.

[11] Maartens, R. (2004) Brane-World Gravity. Living Reviews in Relativity, 7, 7. http://www.livingreviews.org/lrr-2004-7 http://dx.doi.org/10.12942/lrr-2004-7

[12] Rubakov, V. (2002) Classical Theory of Gauge Fields. Princeton University Press, Princeton.

[13] Rubakov, V. (2005) Proceeding of Les Houches Summer School on Theoretical Physics, Session 84: Particle Physics beyond the Standard Model.

[14] Rubakov, V.A. and Tinyakov, P.G. (2008) Infrared-Modified Gravities and Massive Gravitons. Physics-Uspekhi, 51, 759-792. http://arxiv.org/abs/0802.4379 http://dx.doi.org/10.1070/pu2008v051n08abeh006600

[15] Dubovsky, S., Flauger, R., Starobinsky, A. and Tkachev, I. (2010) Signatures of a Graviton Mass in the Cosmic Microwave Background. Report UTTG-06-09, TCC-23- 09, Physical Review, D81, Article ID: 023523. http://arxiv.org/abs/0907.1658 http://dx.doi.org/10.1103/physrevd.81.023523

[16] Alves, M., Miranda, O. and de Araujo, J.J. (2009) Can Massive Gravitons be an Alternative to Dark Energy? http://arxiv.org/abs/0907.5190

[17] Beckwith, A.W. (2015) Deceleration Parameter Q(Z) in Four and Five Dimensional Geometries, and Implications of Gra-Viton Mass In Mimicking De in both Geometries. http://vixra.org/pdf/1002.0056v1.pdf

[18] Beckwith, A.W. (2011) Identifying a Kaluza Klein Treatment of a Graviton. Journal of Cosmology, 13. http://journalofcosmology.com/BeckwithGraviton.pdf

[19] Padmanabhan, T. (2006) An Invitation to Astrophysics. Vol. 8, World Scientific Series in Astronomy and Astrophysics, New York.

[20] Padmanabhan, T. (2000) Theoretical Astrophysics, Volume 1, Astrophysical Processes. Cambridge University Press, Cambridge.

[21] Taveras, V. (2008) Corrections to the Friedmann Equations from LQG for a Universe with a Free Scalar Field. Physical Review, D78, Article ID: 064072. http://arxiv.org/abs/0807.3325 http://dx.doi.org/10.1103/PhysRevD.78.064072

[22] t’Hooft, G. (2006) The Mathematical Basis for Deterministic Quantum Mechanics. http://arxiv.org/PS_cache/quant-ph/pdf/0604/0604008v2.pdf

[23] t’Hooft, G. (2002) Determinism beneath Quantum Mechanics. http://arxiv.org/PS_cache/quant-ph/pdf/0212/0212095v1.pdf

[24] Beckwith, A.W. (2001) Classical and Quantum Models of Density Wave Transport: A Comparative Study. PhD Dissertation, University of Houston, Houston.

[25] Beckwith, A.W. (2006) A New S-S’ Pair Creation Rate Expression Improving upon Zener Curves for I-E Plots. Mod. Physics Letters B, 20, 849-861. http://arxiv.org/abs/math-ph/0411045 http://dx.doi.org/10.1142/s0217984906011219

[26] Peskins, M. (1995) Introduction to Quantum Field Theory. Frontiers in Physics, Advanced Book Program, Perseus Books, Cambridge.

[27] Bashinsky, S. (2005) Coupled Evolution of Primordial Gravity Waves and Relic Neutrinos. http://arxiv.org/abs/astro-ph/0505502

[28] Giovannini, M. (2006) Non Topological Gravitating Defects in 5 Dimensional Space. Classical and Quantum Gravity, 23, L73-L80. http://dx.doi.org/10.1088/0264-9381/23/23/L01

[29] Matarre, S. (2009) Lecture, International School of Astro Particle Physics. Como, Italy.

[30] Raffert, G. (2009) Erice Nuclear Physics School, Private Conversation.

[31] Beckwith, A. (2010) Energy, Neutrino Physics and the Lithium Problem: Why There Are Stars with Essentially No Lithium Due to Serious Lithium Deficiency in Certain Spatial Regions in the Early Universe? Progress in Particle and Nuclear Physics, 64, 426-428. http://dx.doi.org/10.1016/j.ppnp.2009.12.066

[32] Wechsler, R.H. (2001). Dark Halo Merging and Galaxy Formation. PhD Thesis. http://risa.stanford.edu/thesis.php

[33] Eberle, B., Ringwald, A., Song, L. and Weiler, T.J. (2004) Relic Neutrino Absorption Spectroscopy. Report Number DESY 03-219, SLAC-PUB-10302, Physical Review, D70, Article ID: 023007. http://dx.doi.org/10.1103/physrevd.70.023007 http://arxiv.org/abs/hep-ph/0401203

[34] Valev, D. (2008) Neutrino and Graviton Rest Mass Estimations by a Phenomenological Approach. Aerospace Research in Bulgaria, 22, 68-82. http://arxiv.org/abs/hep-ph/0507255 
[35] Beckwith, A. (2010) Applications of Euclidian Snyder Geometry to the Foundations of Space Time Physics. http://vixra.org/abs/0912.0012

[36] Kay, B. (2006) Quantum Field Theory in Curved Spacetime. In: Franciose, J.-P., Naber, G. and Tsun. T., Eds., The Encyclopedia of Mathematical Physics, Vol 5, General Relativity, Quantum Gravity, String Theory and M. Theory, Academic (Elsevier) Publishing Company, Amsterdam, 180-190.

[37] Beckwith, A.W. (2011) Is Nature Fundamentally Continuous or Discrete, and How Can These Two Different but Very Useful Conceptions Be Fully Reconciled? (Condensed Version) http://vixra.org/abs/1102.0019

[38] Padmanabhan, T. (2011) Lessons from Classical Gravity about the Quantum Structure of Spacetime. http://arxiv.org/pdf/1012.4476.pdf

[39] Beckwith, A.W. (2011) How to Use the Cosmological Schwinger Principle for Energy Flux, Entropy, and "Atoms of Space-Time” to Create a Thermodynamic Space-Time and Multiverse. Journal of Physics: Conference Series, 306, Article ID: 012064. http://dx.doi.org/10.1088/1742-6596/306/1/012064 http://iopscience.iop.org/1742-6596/306/1/012064/

[40] Kiefer, C., Polarski, D. and Starobinsky, A.A. (1998) Quantum-to-Classical Transition for Fluctuations in the Early Universe. International Journal of Modern Physics, D7, 455-462. http://arxiv.org/abs/gr-qc/9802003 http://dx.doi.org/10.1142/s0218271898000292

[41] Penrose, R. (2007) Conformal Cyclic Cosmology, Dark Matter, and Black Hole Evaporation. IGC Inaugural Conference, Penn State University, Pennsylvania, 7-11 August2007.

[42] Penrose, R. (2006) Before the Big Bang, an Outrageous New Perspective and Its Implications for Particle Physics. Proceedings of the European Particle Accelerator Conference (EPAC, 2006), 2759-2763,

[43] Penrose, R. (2011) Cycles of Time, an Extrardinary New View of the Universe. Alfred A. Knoff Press, Oxford.

[44] Beckwith, A. (2014) Analyzing Black Hole Super radiance Emission of Particles/Energy from a Black Hole as a Gedankern Experiment to Get Bounds on the Mass of a Graviton. Hindawi Publishing Corporation, Advances in High Energy Physics, 2014, Article ID 230713. http://dx.doi.org/10.1155/2014/230713 http://www.researchgate.net/publication/261709517_httpwww.hindawi.comjournalsahep2014230713

[45] Poplawski, N. (2011) Cosmological Constant from QCD Vacuum and Torsion. Annalen der Physik, 523, 291-295. http://dx.doi.org/10.1002/andp.201000162 http://arxiv.org/abs/1005.0893

[46] Kolb, E. and Turner, M. (1990) The Early Universe, Frontiers in Physics. Advanced Book Program, Addison and Welsley Publishing Company, Menlo Park.

[47] Feynman, R. and Hibbs, A. (2005) Quantum Mechanics and Path Integrals. Dover Publications Inc., New York.

[48] Park, D.K., Kim, H. and Tamarayan. S. (2002) Nonvanishing Cosmological Constant of Flat Universe in Brane World Scenarios. Physics Letters B, 535, 5-10. http://dx.doi.org/10.1016/S0370-2693(02)01729-X

[49] De La Vega, H. (2009) Lecture on Cosmology, and Cosmological Evolution, as Given as a Lecture at the ISAPP (International School of Astro Particle Physics (2009)). Cosmic Microwave Background and Fundamental Interaction Physics, Como, 8-16 July 2009.

[50] Camp, J. and Cornish, N. (2004) Gravitational Wave Astronomy. Annual Review of Nuclear and Particle Science, 54, 525-577. http://dx.doi.org/10.1146/annurev.nucl.54.070103.181251

[51] Grishkuk, L. (2008) Discovering Relic Gravitational Waves in Cosmic Microwave Background Radiation. http://arxiv.org/abs/0707.3319

[52] Tinyakov, P. (2007) Course 12 Giving Mass to the Graviton. In: Bernadeau, F., Grojean, C. and Dalibard. J., Eds., Particle Physics, and Cosmology, the Fabric of Space-Time, Part of Les Houches, Session 86, Elsevier, Oxford, 471499. http://dx.doi.org/10.1016/s0924-8099(07)80038-2

[53] Novello, M. and Neves, R.P. (2003) The Mass of the Graviton and the Cosmological Constant. Classical and Quantum Gravity, 20, L67-L73. http://dx.doi.org/10.1088/0264-9381/20/6/101

[54] Corda, C. (2012) Primordial Gravity’s Breath. http://arxiv.org/pdf/1110.1772

[55] Abbott, B.P., et al. (2016) LIGO Scientific Collaboration and Virgo Collaboration. Physical Review Letters, 116, Article ID: 061102. http://dx.doi.org/10.1103/PhysRevLett.116.061102

[56] The LIGO Scientific Collaboration, the Virgo Collaboration. Tests of General Relativity with GW150914. Physical Review Letters, 116, Article ID: 221101. http://arxiv.org/abs/1602.03841

[57] Corda, C. (2009) Interferometric Detection of Gravitational Waves: The Definitive Test for General Relativity. International Journal of Modern Physics, D18, 2275-2282. http://dx.doi.org/10.1142/s0218271809015904 http://arxiv.org/abs/0905.2502

[58] Kuchiev, M.Y. (1988) Can gravity appear due to polarization of instantons in SO(4)gauge theory?. Classical and Quantum Gravity, 15, 1895-1913. http://dx.doi.org/10.1088/0264-9381/15/7/008 
[59] Haranas, I. and Gkigkitzis, I. (2014) The Mass of Graviton and Its Relation to the Number of Information According to the Holographic Principle. International Scholarly Research Notices, 2014, Article ID: 718251. http://dx.doi.org/10.1155/2014/718251 http://www.hindawi.com/journals/isrn/2014/718251/

\section{Submit or recommend next manuscript to SCIRP and we will provide best service for you:}

Accepting pre-submission inquiries through Email, Facebook, Linkedin, Twitter, etc A wide selection of journals (inclusive of 9 subjects, more than 200 journals) Providing a 24-hour high-quality service User-friendly online submission system Fair and swift peer-review system Efficient typesetting and proofreading procedure Display of the result of downloads and visits, as well as the number of cited articles Maximum dissemination of your research work

Submit your manuscript at: http://papersubmission.scirp.org/ 